\title{
Multi Agent Application for Chronic Patients: Monitoring and Detection of Remote Anomalous Situations
}

\author{
Daniel Hernández ${ }^{(\bowtie)}$, Gabriel Villarrubia, Alberto L. Barriuso, \\ Álvaro Lozano, Jorge Revuelta, and Juan F. De Paz \\ Department of Computer Science and Automation, University of Salamanca, \\ Plaza de la Merced, s/n, 37008 Salamanca, Spain \\ \{danihiglesias, gvg, loza, jrevuelta, fcofds\}@usal.es
}

\begin{abstract}
The clinical study of the most basic vital signs of a patient represents the simplest and most effective way to detect and monitor health problems. There are many diseases that can be diagnosed and controlled through regular monitoring of these medical data. The purpose of this study is to develop a monitoring and tracking system for the various vital signs of a patient. In particular, this work focuses on the design of a multi-agent architecture composed of virtual organizations with capabilities to integrate different medical sensors on an open, low-cost hardware platform. This system integrates hardware and software elements needed for the routine measurement of vital signs, performed by the patient or caregiver without having to go to a medical center.
\end{abstract}

Keywords: WNS $\cdot$ Home care $\cdot$ Healthcare sensors $\cdot$ PANGEA

\section{Introduction}

At present, there are over 860 million people with chronic disease [9]. It is estimated that $25 \%$ of these patients could benefit immediately from solutions for monitoring their health from home [8]; another $50 \%$ would benefit from integration existing medical resources $[11,13]$ into their phones and other devices. In Europe, there is a large group of people who suffer from some type of chronic disease such as diabetes or cardiovascular disease. These patients generate $80 \%$ of healthcare spending in different countries of the European Union [3]. Moreover, they must regularly go to health centers to have their vital signs checked and determine whether they fall within the normal parameters or indicate an abnormality. In many cases, this process involves high costs and inevitable time spent transporting patients from their homes to medical centers, generating long queues, as the medical staff should check the vital signs manually and ensure that they fall within a normal range. In addition, it would be beneficial to monitor certain measurements over a period of time. For example, over a period of one week, an electrocardiogram can collect information about the behavior of the heart during daily physical activity of the patient, which would otherwise not be reproduced in a clinical trial at the medical center [15]. In most cases, the tests are simple and with a little training from medical staff, patients could perform these tests on their own from their 
homes [7]. This training along with appropriate medical tools could help patients maintain a continuous monitoring of their vital signs from the comfort of their homes. This paper presents a virtual architecture with embedded agents that can be assigned to each of the different medical sensors used, using a light communication protocol and a modular architecture that can self-adapt to the particular needs of the patient.

The use of sensor networks and the use of multi-agent systems can develop services that can be interesting and useful to society. Some existing studies, such as [16], proposed a solution based on Context Awareness, deploying a sensor network architecture in nursing homes. In the same context [4] proposes a system based on a multi-agent architecture for managing an intelligent environment nursing home. This paper proposes a system based on virtual organizations of agents deployed on an open hardware and low cost system. It is able to integrate up to nine medical sensors for measuring the vital signs of a user. The PANGEA [17] platform was used for the development of the system, as it facilitates the creation of virtual organizations of agents and their integration into different hardware devices. Specifically, the system integrates different virtual organizations to perform different tasks such as collecting data from various sensors, checking the maximum and minimum range for each collected value, and managing alerts or shipping information to the remote server that will be responsible for storing historical data.

The paper is organized as follows: Sect. 2 includes a collection of related work, Sect. 3 presents the proposed system, and Sect. 4 presents the results and conclusions.

\section{Background}

The current market contains various devices that monitor vital sign by using sensors connected through mobile devices with a wireless connection such as Bluetooth or WiFi. These devices, which include pulse sensors [5], connected glucometers such as the Wireless Blood Glucometer by iHealth, or blood oxygen sensors, are limited to showing the data obtained by the sensor to the user via the mobile device itself, and do not provide any type of information processing. Many studies focus on the development of new medical sensors, as in the case of Dr. Chris Rorden of the American University of South Carolina, who has developed a prototype system ECG (Fig. 1) based on the open source Arduino controller and a Bluetooth system [18].

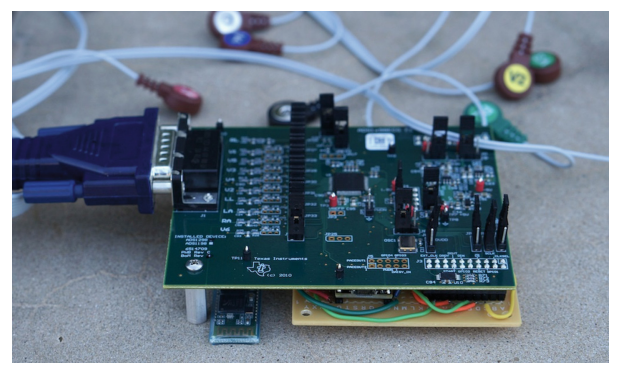

Fig. 1. Prototype of South Carolina's college 
In other works such as [12] the authors have implemented a data analysis system that collects health data from a user's home through the use of various sensors (heart rate, oxygen saturation and body temperature sensor). These sensors send the information wirelessly to the user's mobile phone and use accelerometers placed on the patient to monitor potential falls. The authors in [14] propose an architecture solution based on a wireless sensor network (WSN), focusing their use in a domestic environment and introducing non-intrusive sensors that can analyze the environmental conditions present in the user's daily life. This allows the authors to manage environmental information, which is useful when analyzing the health and potential risk factors in the environment. There are also several works that address the use of sensors and systems for Home Care in nursing homes [4], also called elderly care. Specifically, the authors in [2] stress the current need to create adaptive systems that adapt to the different needs of users.

It is also possible to find works applying multiagent architecture for managing Home Care environments, as in the case of [10]. In this paper the authors propose a multi-agent architecture for the home care of patients with heart failure. The system incorporates multiple processing modules based on statistics, standards and rules that apply to the medical data of patients, as well as learning ability based on previously performed medical procedures. The ultimate goal of this system is to monitor the health status of patients at home and notify medical staff, providing them with the data to assist them in making medical decisions. In [6] the authors analyze the important aspects of context-aware computing and determine how users interact with their environment. The architecture provides a new mechanism that integrates a task planning model based on the critical path method (CPM) [1] in intelligent agents. The CPM method connects a series of related activities in the most optimal way to achieve a specific objective.

\section{Proposed Reasoning System}

The system proposed in this paper aims to monitor and track the user's vital signs. When monitoring this data from the user's home an open and scalable hardware platform based on the Raspberry Pi board [20] was used, on which up to nine medical sensors can be placed. This device is connected to a central server where user data are processed, and also can provide an immediate response if a risk is detected. In order to implement efficient mechanisms to manage the different parameters collected by the medical sensors, and to monitor alerts, sending the data back to a central server, a MAS (multi-agent system) architecture-based on the PANGEA platform [17] was proposed for the Home Care scenario described in Fig. 2.

Then virtual organizations are as follows:

First we have the Measuring Device organization formed by the following agents: alert, monitoring, visualization and sub-organization sensors. This organization is deployed in the hardware system based on Raspberry Pi. The agent monitoring role will be responsible for analyzing the data obtained by the sensor array; if anomalous values are detected, this agent interacts with the agent responsible for managing alerts. The monitoring agent also provides the data to the visualization agent. The agent with the 


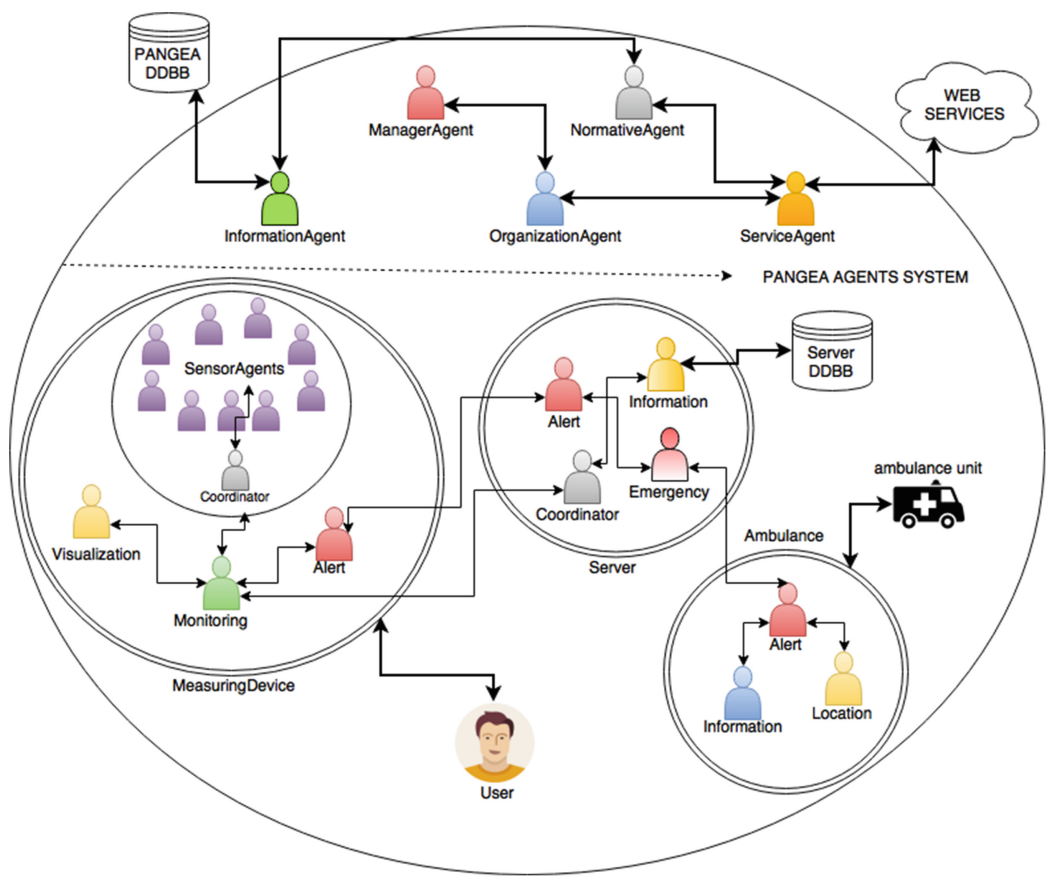

Fig. 2. Virtual organization of the system

alert role is responsible for sending alerts to the server to manage it efficiently. The visualization agent role is responsible for presenting the user data obtained through a monitor connected to the system. The sensor sub-organization includes the coordinator agent, who is responsible for collecting the data sent by each sensor and nine Agents, one for each sensor connected to the system. These agents may or may not be active or depending on whether the sensor is connected to the system.

The Server organization includes the following agents: coordinator, alert, information, and emergency. The coordinator agent role is responsible for managing the information coming from the measuring device. The information agent is responsible for storing user data in the database, while the alert agent analyzes different alerts coming from the measuring device. If these alerts require rapid intervention, the alert agent communicates with the emergency agent, which coordinates the dispatch of the nearest ambulance to the user's home. Finally, the Ambulance organization integrates the following roles: alert, location and information. The agent with the alert role, receives the alert notification from the server. The information relating to this alert will be managed by the information agent, along with the corresponding medical data that initiated the alert, to provide faster response and precise detailing. The information relating to the location and routes to reach the user's home is managed by the location agent. 


\subsection{Sensor System}

The system for measuring the vital signs of the user is capable of integrating up to nine medical sensors, as previously mentioned. The system is able to adapt to the needs of the patient and set the sensors required to monitor their medical problems. There are nine medical sensors that make up the network of biometric sensors; the functionality of each is described below:

Patient Position Sensor: This sensor oversees five different patient positions (standing or sitting, supine, prone, left and right). In many cases, it is necessary to control body positions and movements performed because of a relationship with chronic diseases (sleep apnea or restless legs syndrome). Analyzing the movements during sleep can also help determine the quality of sleep and irregular sleep patterns.

Glucose Sensor or Glucometer: A medical device to determine the approximate concentration of glucose in the blood. A small drop of blood, obtained by pricking the skin with a lancet, is placed on a disposable test strip which is read by the meter and used to calculate the blood glucose level. The meter then displays the level in $\mathrm{mg} / \mathrm{dl}$ or $\mathrm{mmol} / \mathrm{l}$.

Temperature Sensor or Thermometer: This sensor can measure body temperature. This data is of great medical importance as it can quickly determine if there are any health problems. In fact, a number of diseases are accompanied by characteristic changes in body temperature. Likewise, the course of certain diseases can be monitored by measuring body temperature, and the efficiency of a treatment initiated can be evaluated by the physician.

Blood Pressure Sensor: Blood pressure is the pressure of the blood in the arteries as it is pumped around the body by the heart. When the heart is beating, it contracts and pushes blood through the arteries to the rest of the body. This force creates pressure on the arteries. Blood pressure is recorded as two numbers - the systolic pressure (as the heart beats) over the diastolic pressure (as the heart relaxes between beats).

Pulse and Oxygen in Blood: Oxygen saturation is defined as the measurement of the amount of oxygen dissolved in the blood, based on the detection of Hemoglobin and Deoxyhemoglobin. Two different light wavelengths are used to measure the actual difference in the absorption spectra of $\mathrm{HbO} 2$ and $\mathrm{Hb}$.

Airflow Sensor: Abnormal respiratory rates and changes in respiratory rate are a broad indicator of major physiological instabilities, and in many cases, respiratory rate is one of the earliest indicators of this instability. Therefore, it is critical to monitor respiratory rate as an indicator of patient status. AirFlow sensor can provide an early warning of hypoxemia and apnea.

Sensor Skin Conductance: Skin conductance, also known as galvanic skin response (GSR), is a method of measuring the electrical conductance of the skin, which varies according to its moisture level. This is significant because sweat glands are controlled by the sympathetic nervous system, so moments of strong emotion change the electrical resistance of the skin. Skin conductance is used as an indication of psychological or physiological arousal. 
Electrocardiogram Sensor (ECG): The electrocardiogram (ECG or EKG) is a diagnostic tool that is routinely used to assess the electrical and muscular functions of the heart. The Electrocardiogram Sensor (ECG) has become one of the most commonly used medical tests in modern medicine. Its utility in the diagnosis of a myriad of cardiac pathologies ranging from myocardial ischemia and infarction to syncope and palpitations has been invaluable to clinicians for decades.

Sensor Electromiógrafo: An electromyogram (EMG) measures the electrical activity of muscles at rest and during contraction. Electromyography (EMG) is a technique for evaluating and recording the electrical activity produced by skeletal muscles. It can be used to identify neuromuscular disorders, and to evaluate lower back pain, motor system disorders, and kinesiology. An EMG is performed by using an instrument called an electromyograph to produce a record called an electromyogram. It detects the electrical potential generated by muscle cells when these cells are electrically or neurologically activated.

Each of the sensors that make up the architecture are low cost (less than $300 €$ per set). The chosen sensors are flexible and adaptable to the needs of the patient. The early-warning system and patient monitoring is described below.

\subsection{Monitoring and Alert System}

The alarm system is based on the detection of any anomalous values collected by any of the sensors that make up the platform. The system considers any value to be anomalous if it is outside normal limits previously defined in the system. These limits must be defined by a medical professional for each patient because the configuration can be different. Some of the elements that must be taken into consideration when defining these values are age, sex or weight. But these are not the only data that must be taken into account; to begin, it is essential to analyze the patient's health problems efficiently in order to set the normal range of data collected from each sensor and properly determine the alerts. For a healthy adult, without previous health problems, the range of correct values is described in Table 1.

Table 1. Normal levels of a user

\begin{tabular}{l|l|l}
\hline Sensor & Minimum value & Maximum value \\
\hline Glucometer & $70 \mathrm{mg} / \mathrm{dl}$ & $100 \mathrm{mg} / \mathrm{dl}$ \\
\hline Thermometer & $36.5{ }^{\circ} \mathrm{C}$ & $37 .{ }^{\circ} \mathrm{C}$ \\
\hline Blood pressure & $100 / 60 \mathrm{mmHg}$ & $140 / 90 \mathrm{mmHg}$ \\
\hline Pulsometer & $60 \mathrm{BPM}$ & $100 \mathrm{BPM}$ \\
\hline Oximeter & $96 \% \mathrm{O}^{2}$ in blood & $99 \% \mathrm{O}^{2}$ in blood \\
\hline Nasal airflow & $12 \mathrm{breath} / \mathrm{min}$ & $30 \mathrm{breath} / \mathrm{min}$ \\
\hline Skin conductance & $1 \mu \mathrm{S}$ & $10 \mu \mathrm{S}$ \\
\hline
\end{tabular}

In the case of the values given by the electrocardiogram and the electromyography, it is necessary to compare the values collected by these sensors in normal conditions to then determine the degree of difference from the monitored values to determine whether to launch an alert. 


\subsection{Alert System for Ambulances}

The central server is responsible for evaluating the alerts that have been sent by the measurement system in the user's home (Fig. 3). An alert occurs if, while measuring the user's vital signs, the system records a value that has been marked outside the "normal" range for that user. This value, which is sent to the central server, is compared with the patient's historical data. If the data sent are not compatible with those on record, the system determines that it is an emergency. When this occurs, the system sends a notification to the ambulance nearest to the home user. Ambulances associated with the system have a mobile device with the emergencies application installed. This application receives geolocation information from home user and calculates the shortest route to get there.

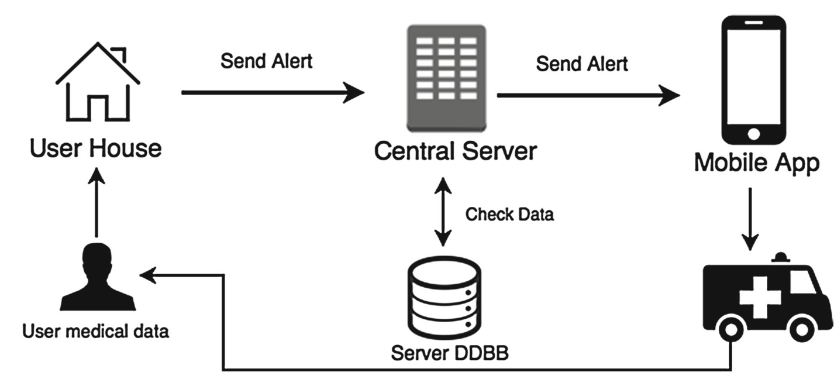

Fig. 3. Alerta sistem

\section{Results and Conclusions}

In order to validate the system, we selected a home in the town of Macotera (Salamanca, Spain) where a user voluntarily tested the system for 45 days. The user is a man of 25 years with heart problems who takes his vital signs twice daily. Figure 4 shows the user with various sensors placed on his person while performing a measurement and monitoring the results from a display connected to the device. The medical sensors included in the prototype are shown in Fig. 5, these sensors are manufactured by the company Cooking-hacks [19].

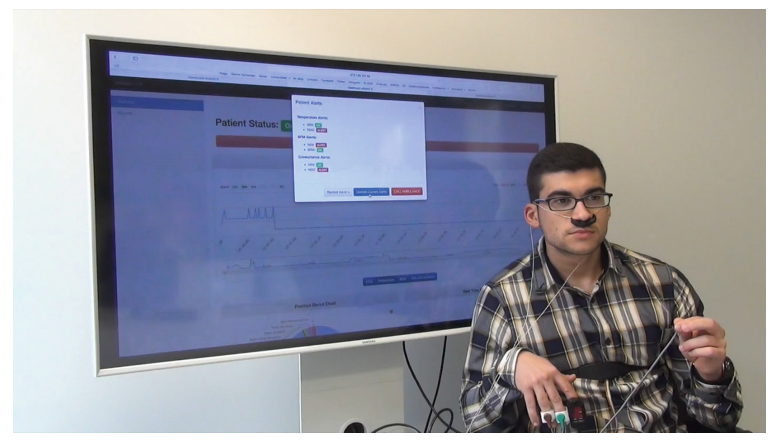

Fig. 4. Real user testing the system 


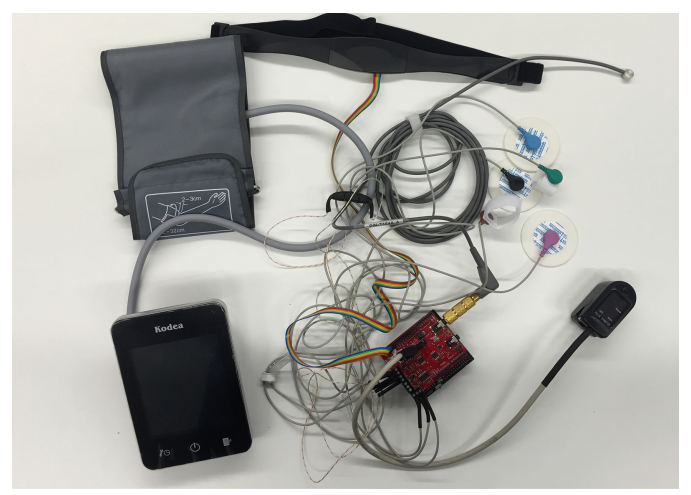

Fig. 5. Medical sensors

If any of the data recorded in the measurement fall outside the normal ranges established by the user, with the assistance of a medical professional, the system sounds an alarm and informs the user through screen, as seen in Fig. 6.

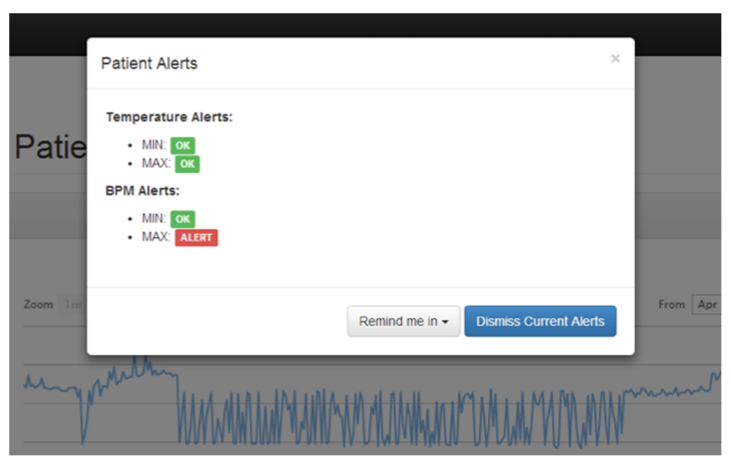

Fig. 6. Patient Alerts

While the user is notified of the alert, the notification arrives at the central server where it is analyzed together with the historical patient data. Having determined an emergency, the server proceeds to notify the nearest ambulance of the incident. The ambulance is notified of the alert via the mobile application installed. Figure 7(a) shows how the emergency notification arrives on the mobile device of an ambulance. In Fig. 7(b) we can observe how the notification includes the location coordinates of the patient as well as the shortest route to arrive.

In conclusion, this paper has proposed a multi-agent system based on the PANGEA platform to manage different medical data system. These data are captured by a hardware device equipped with nine medical sensors able to monitor a user's basic vital signs from home. This system also connects to a central server which manages and 


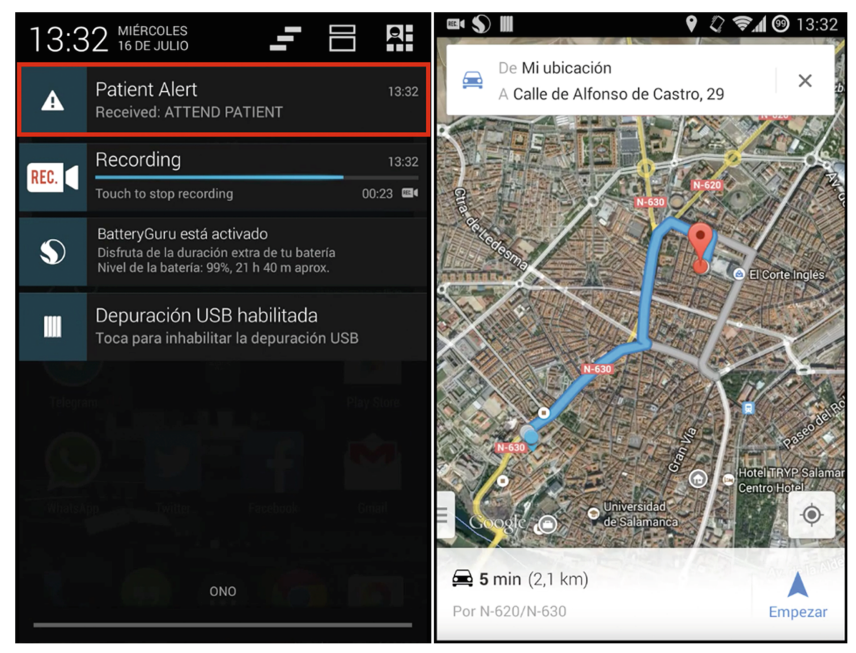

Fig. 7. Ambulance App. (a) Alert notification. (b) Route.

stores historical data, and serves as an emergency center in case of an anomaly in the data. The main advantages of the proposed system are:

- The low cost of the sensors.

- The analysis of the information for each patient, which can help predict a chronic illness well in advance.

- Save on travel time and eliminate different human errors that can occur when reading values.

- Monitor the patient $24 \mathrm{~h}$ and know their status at all times.

- Simultaneous monitoring of up to 9 vital signs.

- Ability to access all user tests and results from anywhere.

- Emergency system to detect problems in the data.

Acknowledgements. The research of Alberto L. Barriuso has been co-financed by the European Social Fund (Operational Programme 2014-2020 for Castilla y León, EDU/128/2015 BOCYL).

\section{References}

1. Aquilano, N.J., Smith, D.E.: A formal set of algorithms for project scheduling with critical path scheduling/material requirements planning. J. Oper. Manage. 1(2), 57-67 (1980)

2. van den Berg, N., et al.: Telemedicine and telecare for older patients-a systematic review. Maturitas 73(2), 94-114 (2012)

3. Busse, R., et al.: Tackling chronic disease in Europe: strategies, interventions and challenges. Obs. Stud. 20, 111 (2010)

4. Corchado, J.M., et al.: GerAmi: improving healthcare delivery in geriatric residences. IEEE Intell. Syst. 23(2), 19-25 (2008) 
5. Fezari, M., et al.: Ambulatory health monitoring system using wireless sensors node. Procedia Comput. Sci. 65, 86-94 (2015)

6. Fraile, J.A. et al.: Context-aware multiagent system: planning home care tasks. Knowl. Inf. Syst. (2013)

7. Howe, A., Anderson, J.: Involving patients in medical education. BMJ 327(7410), 326-328 (2003)

8. Huang, F.-H.: Explore home care needs and satisfaction for elderly people with chronic disease and their family members. Procedia Manuf. 3, 173-179 (2015)

9. Knai, C., et al.: Systematic review of the methodological quality of clinical guideline development for the management of chronic disease in Europe. Health Policy 107(2-3), 157-167 (2012)

10. Koutkias, V.G., et al.: Multi-agent system architecture for heart failure management in a home care environment. Comput. Cardiol. 2003, 383-386 (2003)

11. Preuveneers, D., et al.: The future of mobile e-health application development: exploring HTML5 for context-aware diabetes monitoring. Procedia Comput. Sci. 21, 351-359 (2013)

12. Varma, D., et al.: Development of home health care self monitoring system 4(6), 252-255 (2015)

13. Varshney, U.: Mobile health: Four emerging themes of research. Decis. Support Syst. 66, 20-35 (2014)

14. Virone, G., et al.: An advanced wireless sensor network for health monitoring. Transdiscipl. Conf. Distrib. Diagnosis Home Healthc. 2-5 (2006)

15. Wimmer, N.J., et al.: The clinical significance of continuous ECG (ambulatory ECG or Holter) monitoring of the ST-segment to evaluate ischemia: a review. Prog. Cardiovasc. Dis. 56(2), 195-202 (2013)

16. Wood, A., et al.: Context-aware wireless sensor networks for assisted living and residential monitoring. IEEE Netw. 22(4), 26-33 (2008)

17. Zato, C., et al.: PANGEA: a new platform for developing virtual organizations of agents. Int. J. Artif. Intell. 11(13A), 93-102 (2013)

18. Chris Rorden's Neuropsychology Lab » Open source multi-channel EEG/ECG/EMG. http://www.mccauslandcenter.sc.edu/CRNL/tools/ads1298

19. Cooking Hacks - Electronic Kits, tutorials and guides for Makers and Education. https:// www.cooking-hacks.com/

20. Raspberry Pi - Teach, Learn, and Make with Raspberry Pi. https://www.raspberrypi.org/ 